\title{
Ectoparasitos em preás (Galea spixii Wagler, 1831) cativos no semiárido do Rio Grande do Norte $^{1}$
}

\author{
Josivania S. Pereira ${ }^{2 *}$, Leonardo C.A. Carvalhoº ${ }^{2}$, Benito Soto-Blanco ${ }^{2}$, Moacir F. Oliveira ${ }^{2}$ \\ e Sílvia M.M. Ahid ${ }^{2}$
}

\begin{abstract}
Pereira J.S., Carvalho L.C.A., Soto-Blanco B., Oliveira M.F. \& Ahid S.M.M. 2012. [Ectoparasites in guinea pigs (Galea spixii Wagler, 1831) in captivity in the semiarid region of Rio Grande do Norte, Brazil.] Ectoparasitos em preás (Galea spixii Wagler, 1831) cativos no semiárido do Rio Grande do Norte. Pesquisa Veterinária Brasileira 32(8):789793. Departamento de Ciências Animais, Universidade Federal Rural do Semi-Árido, Avenida Francisco Mota 572, Bairro Presidente Costa e Silva, Mossoró, RN 59625-900, Brazil. E-mail: josigej@ufersa.edu.br

Wild rodents in captivity can be attacked by ectoparasites which affect their health. This study aimed to identify the natural ectofauna of the guinea pig Galea spixii Wagler, 1831 in captivity in the semiarid region of Rio Grande do Norte and to study the behavioral dynamics of the most prevalent ectoparasitism. Specimens $(\mathrm{n}=40)$ of $G$. spixii in captivity in the Center for Wild Animal Multiplication (Centro de Multiplicação de Animais Silvestres da Universidade Federal Rural do Semi-Árido) were anesthetized from March to October 2010 and examined searching for ectoparasites. The guinea pigs presented Amblyomma sp. (Koch, 1844), Demodex sp. (Owen, 1843) and Gliricola quadrisetosa (Ewing, 1924). The frequency of data of $G$. quadrisetosa, the most prevalent species in the study, showed that the mean of lice collected in the different body parts was higher in females $(\mathrm{p}=0,0498)$. The climatic period did not influence the frequency of $G$. quadrisetosa collected from the animals ( $p>0.05)$. Demodex sp. was the first record of ectofauna identified in G. spixii in semiarid conditions in Brazil. The data indicate that the body surface area and the climatic period did not influence the infra-population of Gliricola quadrisetosa found in Galea spixii.
\end{abstract}

INDEX TERMS: Galea spixii, Amblyomma sp., Demodex sp., Gliricola quadrisetosa.

RESUMO.- Os roedores silvestres quando criados em cativeiro podem ser acometidos por ectoparasitos que afetam a sua sanidade. Este trabalho objetivou identificar a ectofauna natural do preá Galea spixii criado nas condições de cativeiro no semiárido do Rio Grande do Norte e estudar o comportamento da dinâmica comportamental do ectoparasitismo mais prevalente. Utilizou-se 40 espécimes de G. spixii cativos do Centro de Multiplicação de Animais Silvestres da Universidade Federal Rural do Semi-Árido. Os exemplares foram anestesiados e examinados para busca de ectoparasitos, durante os meses de março a outubro de 2010. Os preás estudados apresentaram Amblyomma sp.,

\footnotetext{
${ }^{1}$ Recebido em 20 de agosto de 2011.

Aceito para publicaçãpo em 4 de maio de 2012.

${ }^{2}$ Departamento de Ciências Animais, Universidade Federal Rural do Semi-Árido (UFERSA), Avenida Francisco Mota 572, Bairro Presidente Costa e Silva, Mossoró, RN 59625-900, Brasil. *Autor para correspondência: josigej@ufersa.edu.br
}

Demodex sp. e Gliricola quadrisetosa. Os dados de frequência de G. quadrisetosa, espécie de maior prevalência, revelaram que a média de piolhos recuperados nas distintas áreas corporais, foi maior para exemplares fêmeas ( $p=0,0498)$. 0 período climático não influenciou na frequência de G. quadrisetosa recuperada dos animais $(\mathrm{p}>0,05)$. Da ectofauna identificada em G. spixii, notifica-se Demodex sp. como primeiro registro neste roedor nas condições semiáridas do Brasil. Os dados sugerem que a área corporal e o período não interferiram na infra população de Gliricola quadrisetosa encontrada em Galea spixii.

TERMOS DE INDEXAÇÃo: Galea spixii, Amblyomma sp., Demodex sp., Gliricola quadrisetosa.

\section{INTRODUÇÃo}

Os pequenos mamíferos constituem um grupo ecológico economicamente importante, tanto do ponto de vista da abundância e diversidade de espécies, quanto por serem 
encontrados como componentes fundamentais em quase todos os ecossistemas terrestres. Nos últimos anos, tem-se notado um aumento na criação desses animais considerados fonte de proteína natural, principalmente nas regiões tropicais e subtropicais do nosso planeta (Reis et al. 2008).

Muitos desses animais, principalmente mamíferos roedores, são criados em cativeiros não apenas com o objetivo de fornecer alimento, mas para manter a espécie em seus habitats naturais, procurando uma alternativa para sua conservação e assim proporcionar o desenvolvimento de pesquisas voltadas ao conhecimento mais aprofundado destes organismos (Lacerda et al. 2006).

O preá, Galea spixii Wagler, 1831, é um pequeno roedor encontrado em todos os estados da região Nordeste do Brasil, e também em Minas Gerais e Mato Grosso (Moojen 1952). Pertence à subordem Hystricognathi, família Caviidae e subfamília Caviinae. É um animal silvestre cujas características são: ausência de cauda, superfície dorsal acinzentada e ventral branca, com manchas infraoculares e pós-auriculares brancas e morfologicamente semelhantes às demais espécies da subfamília Caviinae (Lacher 1981).

Quando em cativeiro, os preás são acometidos por doenças de natureza infecciosa ou parasitária. Os mesmos atuam como fontes de infecção para insetos vetores de endoparasitos (Barbosa 2005, Barbosa et al. 2008). A espécie G. spixii pode sofrer ação de ectoparasitos, dentre eles, pulgas, carrapatos, ácaros e piolhos. Estes interferem na saúde e bem-estar deste cavídeo por ocasionar prurido intenso, alopecia e crostas cutâneas na pele, predispondo a infecção bacteriana secundária (Ahid et al. 2009).

Considerando os ectoparasitos como um dos principais problemas que afetam a sanidade de roedores, unido a escassez de dados na literatura relacionados à biologia e ectoparasitismo dos mesmos, teve-se como objetivo a identificação da ectofauna natural de $G$. spixii mantido em regime de cativeiro, no estado do Rio Grande do Norte, Brasil e o estudo da dinâmica comportamental do ectoparasitismo mais prevalente.

\section{MATERIAL E MÉTODOS}

Foram utilizados machos adultos de Galea spixii (N=40) (Fig.1A), cativos do Centro de Multiplicação de Animais Silvestres (CEMAS) da Universidade Federal Rural do Semi-Árido (UFERSA), localizado em Mossoró, Rio Grande do Norte (05¹1'15'S e 37²0'39”W), sob autorização no 1478912 pelo Instituto Brasileiro do Meio Ambiente e dos Recursos Naturais Renováveis (IBAMA). Estes hospedeiros foram identificados através da morfologia e com base em bibliografia específica consultada para roedores.

Os cavídeos, individualmente, foram submetidos a procedimentos de coleta, cuja metodologia utilizada, seguiu as normas de ética com o uso de animais e foi aprovada pelo Comitê de Ética no uso de animais em pesquisa da UFERSA sob parecer $\mathrm{n}^{\mathrm{o}}$ 23091.000335/2011-12.

Os ectoparasitos foram coletados dos animais em dois períodos distintos: de chuva, representado pelos meses de março a abril de 2010, com respectiva temperatura média do ar em torno de $27,94^{\circ} \mathrm{C}$; e precipitação pluviométrica $168,05 \mathrm{~mm}$; e de seca, onde considerou-se os meses de setembro a outubro de 2010, os quais apresentaram temperatura média do ar de $28,14^{\circ} \mathrm{C}$; e precipitação pluviométrica de $7,9 \mathrm{~mm}$, respectivamente.
Para remoção dos ectoparasitos, os espécimes de G. spixii foram contidos quimicamente (Xilazina $10 \%$ e Cetamina $5 \%$, nas doses respectivas de $3 \mathrm{mg} / \mathrm{kg}$ e $15 \mathrm{mg} / \mathrm{kg}$ ). Individualmente, toda a superfície corporal foi inspecionada. Quando detectado carrapatos, estes eram coletados por giro sobre o seu eixo para evitar a perda de estruturas do gnatossoma.

Realizou-se raspados cutâneos da área posterior dorsal e inferior da mandíbula dos preás, bem como obtenção de cerúmen dos dois pavilhões auriculares com auxílio de swabs. 0 material resultante destes procedimentos foi transferido para lâmina, clareado em solução de hidróxido de potássio a $10 \%$, por tempo médio de 3 horas e analisado através de microscopia óptica (aumento de 10x, 40x e 100x).

Para o penteamento com pente fino das áreas corporais dorsal e lateral utilizou-se as delimitações segundo Bittencourt et al. (2002). 0 material obtido da escovação era colocado em placas de Petri contendo álcool a 70\% e levado ao estereomicroscópio para análise.

Posteriormente cada cavídeo foi banhado em água adicionada de detergente neutro (10\%), por tempo médio de um minuto. Em seguida, a solução do banho contendo sujidades provenientes do corpo do animal foi tamisada para coleta dos ectoparasitas. Estes últimos foram processados no Laboratório de Parasitologia Animal da UFERSA.

Os espécimes recuperados foram identificados através de chaves dicotômicas segundo Werneck $(1936,1942)$, Aragão \& Fonseca (1961) e Barros-Battesti et al. (2006), com auxílio da microscopia óptica e estereomicroscopia.

Os dados obtidos foram apresentados na forma de média simples e erro-padrão. A comparação estatística foi realizada por meio do Teste $t$, para os dados paramétricos, e Teste de Mann-Whitney, para os dados não paramétricos. 0 nível de significância foi estabelecido como $\mathrm{p}<0,05$. As análises foram realizadas com auxílio do programa BioEstat 5.0.

\section{RESULTADOS}

Todos os ectoparasitos recuperados dos animais experimentais foram identificados como Amblyomma sp. (Koch 1844), Demodex sp. e Gliricola quadrisetosa (Ewing 1924).

Da inspeção realizada nos preás, observou-se que nove (22,5\%) apresentaram parasitismo por larvas de Amblyomma sp. na região do pavilhão auricular e periocular, identificadas segundo as características morfológicas: sulco anal posterior ao ânus, olhos presentes e de palpos mais longos do que largos, com o segundo artículo mais do que duas vezes o comprimento do terceiro.

Dos espécimes submetidos ao raspado cutâneo da área dorsal, um $(2,5 \%)$ apresentou positividade para ácaros do gênero Demodex sp. Para os raspados cutâneos da área inferior da mandíbula e swabs dos pavilhões auriculares, verificou-se positividade para este Demodecidae em 14 (35\%) e um $(2,5 \%)$ dos cavídeos analisados, respectivamente.

Os ácaros Demodex sp. foram observados em diferentes estádios evolutivos e identificados pelo corpo vermiforme, abdome alongado, estriado transversalmente e adultos com quatro pares de patas rudimentares. Nas fêmeas o orifício genital é ventral, em fenda longitudinal e situado ao nível da coxa IV (Fig.1B).

No procedimento de penteamento das áreas dorsal e lateral dos roedores, observou-se que todos apresentaram infestação por Phthiraptera, especificamente, Glirico- 

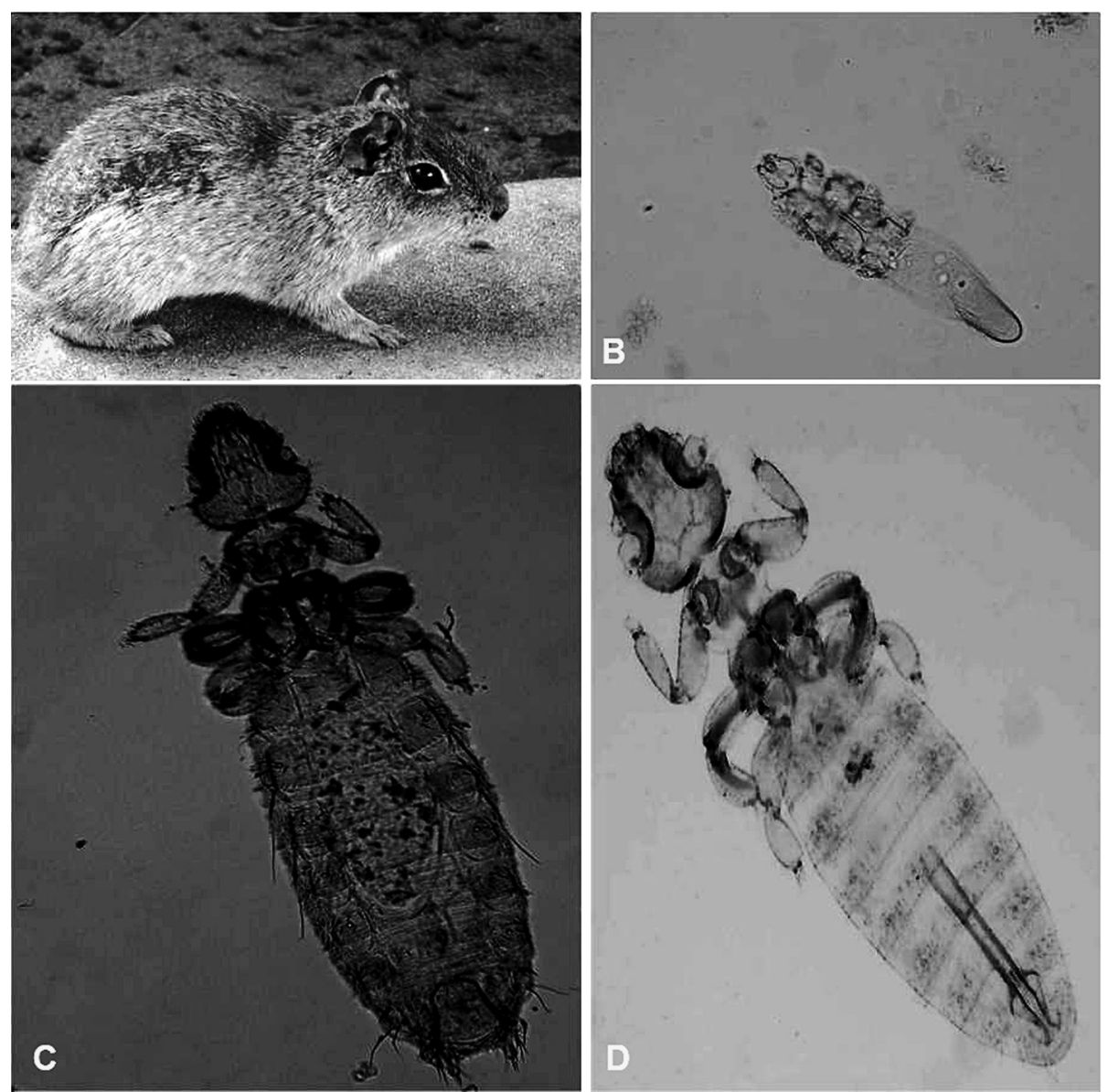

Fig.1. (A) Exemplar de Galea spixii nas condições de estudo. (B) Demodex sp., vista ventral da fêmea, 40x. Gliricola quadrisetosa em vista ventral: (C) fêmea, 10x, (D) macho, 10x.

la quadrisetosa. As amostras resultantes do banho estavam igualmente positivas para esta espécie. Deste último, recuperou-se 8.153 exemplares de G. quadrisetosa.

Este Amblycera recuperado de G. spixii foi identificado segundo as seguintes características taxonômicas: protórax arredondado; meso e metatórax reunidos, formando um só segmento, o pterotórax; abdome com margens posteriores dotadas de fileiras regulares de cerdas pequenas, além destas cerdas curtas, acha-se outras longas, em menor número, dispostas irregularmente e abdome com cinco pares de estigmas respiratórios (Fig.1C).

A confirmação dos espécimes fêmeas de G. quadrisetosa foi dada pela presença de um par de longas cerdas no $2^{\circ}$ e $3^{\circ}$ pleuritos abdominais, característica marcante e única desta espécie, e genitália formada de gonopodos grandes e salientes na extremidade posterior do abdômen (Fig.1C). Os machos, pela ausência das longas cerdas nos pleuritos mencionados e por possuir genitália composta de placa basal longa, cuja extremidade posterior apresenta dois grandes ramos terminais (externo e interno) fortemente encurvados para fora (Fig.1D).

Através do penteamento das áreas dorsal e lateral dos 40 animais experimentais obteve-se 13.629 piolhos da espécie G. quadrisetosa. Ao correlacionar a frequência deste Amblycera em relação à área corporal, observou-se que não existe diferença estatística significativa $(p>0,05)$ entre à média do número de piolhos adultos relacionada com a média de ninfas obtidas do dorso dos mesmos. Dados similares foram registrados ao comparar à média do número de piolhos adultos com a média de ninfas na área corporal lateral dos preás (Quadro 1).

Não houve diferença estatística significativa ao relacionar a média de piolhos do sexo fêmea em relação a macho, coletados do dorso e lateral dos cavídeos (Quadro 1). Diferença estatística significativa $(p=0,0498)$ foi registrada quando comparado a média de exemplares fêmeas em relação aos machos, nas mesmas regiões corporais (dorso + lateral) (Fig.2)

Quadro 1. Médias e erros padrão de Gliricola quadrisetosa, em relação à área corporal de Galea spixii $(n=40)$

\begin{tabular}{lccccc}
\hline Área corpórea & Fase de vida & $\mathrm{n}$ & Média $\pm \mathrm{EP}$ & $\mathrm{CV}$ & Valor $\mathrm{p}$ \\
\hline \multirow{2}{*}{ Dorso } & Ninfa & 4.057 & $101,42 \pm 14,63$ & 91,15 & 0,2092 \\
& Adultos & 2.938 & $73,45 \pm 8,70$ & 74,95 & \\
& Macho & 1.250 & $31,25 \pm 3,71$ & 75,15 & 0,1718 \\
& Fêmea & 1.688 & $42,20 \pm 5,16$ & 77,36 & \\
Lateral & Total & 6.995 & $174,87 \pm 22,64$ & 81,85 & \\
& Ninfa & 4.203 & $105,07 \pm 17,15$ & 103,1 & 0,1764 \\
& Adultos & 2.431 & $60,77 \pm 6,80$ & 70,75 & \\
& Macho & 1.079 & $26,97 \pm 3,32$ & 77,92 & 0,1572 \\
& Fêmea & 1.352 & $33,80 \pm 3,67$ & 68,71 & \\
& Total & 6.634 & $165,85 \pm 22,59$ & 86,09 &
\end{tabular}

$\overline{\mathrm{EP}}=$ Erro padrão, $\mathrm{CV}=$ Coeficiente de variação. ${ }^{*}$ Significa diferença estatística $(\mathrm{P}<0,05)$. 


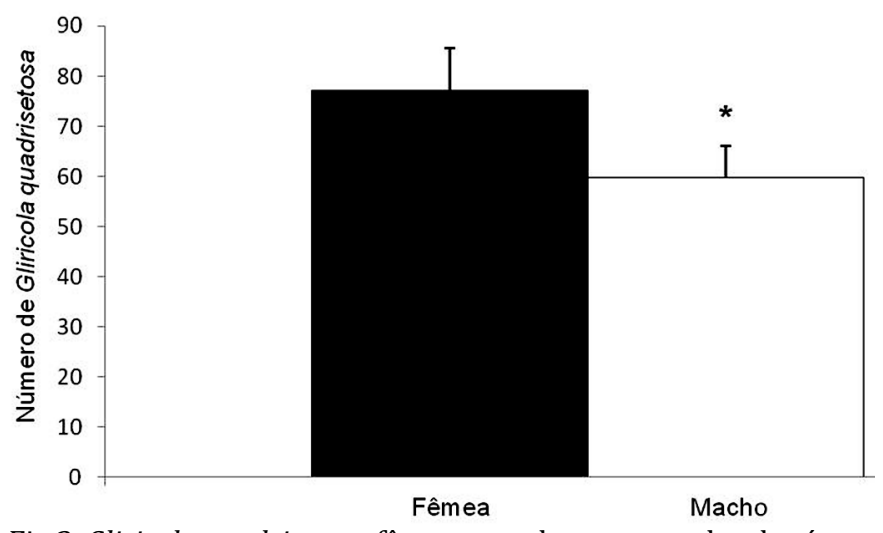

Fig.2. Gliricola quadrisetosa fêmea e macho recuperados das áreas corporais dorsal e lateral de Galea spixii $(\mathrm{N}=40)$. 0 asterisco significa a diferença estatística $(\mathrm{p}<0,05)$.

Quando se correlacionou a frequência de G. quadrisetosa em relação ao período climático, observou-se não haver diferença estatística entre a média de piolhos coletados nas distintas áreas corporais e nos períodos estudados (Quadro 2).

Ao relacionar a média de piolhos coletados na área corporal dorsal dos animais nos dois períodos climáticos, observou-se que esses dados não apresentaram diferença estatística significativa. 0 mesmo foi considerado para a região lateral (Quadro 2).

Ao correlacionar a média de piolhos adultos recuperados no dorso e lateral dos animais e no período de chuva, em relação a média obtida no período de seca, não foi observado diferença estatística significativa entre as mesmas. Dados similares foi registrado ao considerar a fase de ninfa destes ectoparasitos (Quadro 2).

Quadro 2. Médias e erros padrão de Gliricola quadrisetosa, segundo área corporal de Galea spixii $(\mathrm{n}=20)$, submetidos a diferentes condições climáticas

\begin{tabular}{|c|c|c|c|c|c|c|}
\hline \multirow{3}{*}{$\begin{array}{c}\text { Área cor- } \\
\text { poral }\end{array}$} & \multirow{3}{*}{$\begin{array}{c}\text { Fase de } \\
\text { vida }\end{array}$} & \multicolumn{4}{|c|}{ Período climático } & \multirow[t]{3}{*}{ Valor $\mathrm{p}$} \\
\hline & & \multicolumn{2}{|l|}{ Chuva } & \multicolumn{2}{|l|}{ Seca } & \\
\hline & & Média + EP & $\mathrm{CV}$ & Média \pm EP & $\mathrm{CV}$ & \\
\hline \multirow[t]{3}{*}{ Dorso } & Ninfa & $\pm 11,58$ & 63,21 & $120,95 \pm 26,52$ & 98,04 & 0,5792 \\
\hline & Adu & 1,57 & 74,36 & 13,69 & 79,10 & 0 \\
\hline & Total & $151,45 \pm 22,37$ & 66,05 & $198,30 \pm 39,63$ & 89,36 & 0,6750 \\
\hline \multirow[t]{3}{*}{ Lateral } & Ninfa & $81,50 \pm 16,92$ & 92,81 & $128,65 \pm 29,36$ & 102,0 & 0,3040 \\
\hline & Adulto & $59,55 \pm 11,08$ & 83,22 & $62,00 \pm 8,34$ & 60,15 & 0,5609 \\
\hline & Total & $141,05 \pm 27,31$ & 86,58 & $190,65 \pm 36,71$ & 86,08 & 0,2850 \\
\hline
\end{tabular}

$\overline{\mathrm{EP}}$ = Erro padrão, $\mathrm{CV}=$ Coeficiente de variação. ${ }^{*}$ Significa diferença estatística $(\mathrm{P}<0,05)$.

\section{DISCUSSÃO}

Da ectofauna identificada no cavídeos estudados notifica-se Demodex sp. como primeiro registro de um Demodecidae acometendo regiões corporais (pavilhão auricular, dorso e parte inferior da mandíbula) da espécie Galea spixii criado em condições de cativeiro, no Brasil.

O parasitismo por Demodex sp. em roedores não ocorre apenas nas áreas corporais estudadas no presente trabalho, mas em outras regiões, dentre elas: superfícies da genitália, focinho, dorso da cabeça (Nutting 1961), glândulas prepucial, clitorial (Bukva 1985) e membros posteriores (Schönfelder et al. 2010).
Os dados do presente estudo referente ao parasitismo no pavilhão auricular para Demodex sp. reforçam os achados de Bukva (1995), que observou este mesmo ácaro em roedores da espécie Rattus norvegicus, no sul da Bohemia, República Checa, e Bukva (1994) que confirmou este Demodecidae em Apodemus agrarius, na Slovakia.

Na região dorso-sacral de roedores foi igualmente notificada a presença de Demodex sp. por Hasegawa (1995) no Japão em Mesocricetus auratus e por Bukva (1995) na República Checa, em R. Norvegicus.

A cerca do parasitismo por larvas de Amblyomma sp. em G. spixii, os dados do presente trabalho corrobora com o observado por Carvalho et al. (2010) e reforçam os achados de Nava et al. (2008), Nava et al. (2006) que observaram em roedores infestação por este Ixodidae na fase larvar.

Infestação por Gliricola quadrisetosa em Galea spixii foi anteriormente descrita por Werneck (1942) e recentemente por Ahid et al. (2009). Apesar de a presente pesquisa mostrar o parasitismo apenas para este Amblycera, Werneck (1942) relata outro Phthiraptera, mas especificamente Heterogyropus heteronychus, como piolho mastigador específico deste cavídeo.

Werneck (1942) relatou que G. quadrisetosa é um Amblycera que parasita especificamente o roedor $G$. spixii, o que diverge do observado por Werneck (1936) que verificou a ocorrência desta espécie em outros cavídeos, dentre eles: Cavia porcellus e Cavia aperea.

Os dados do presente estudo revelaram que no roedor estudado não existe preferência por superfície corporal para a ectofauna de Phthiraptera identificada, divergindo de Bittencourt \& Rocha (2002), cuja ectofauna encontrada em roedores, especificamente Proechimys iheringi Thomas, 1911 (Echymyidae), no Brasil, em especial o gênero Gliricola apresentou preferência por algumas áreas espécifica do corpo do hospedeiro, dentre elas o dorso e pescoço.

\section{CONCLUSÕES}

Os ectoparasitos naturais identificados em Galea spixii criado em condições de cativeiro em Mossoró, Rio Grande do Norte, foram: Amblyomma sp., Demodex sp. e Gliricola quadrisetosa.

Gliricola quadrisetosa, ectoparasito de maior prevalência, parasitou G. spixii submetidos às condições do presente trabalho, independentemente da área corporal.

O período climático não influenciou na infra população de Gliricola quadrisetosa recuperada em G. spixii.

\section{REFERÊNCIAS}

Ahid S.M.M., Moura G.H.F., Filgueira K.D. \& Oliveira M.F. 2009. Parasitismo por Phthiraptera em preás (Galea spixii spixii) cativos no semiárido do nordeste brasileiro. $2^{\circ}$ Encontro Internacional da Conservação, Recife, PE. (Resumo)

Aragão H. \& Fonseca F. 1961. Notas de Ixodologia. VIII. Chave para famílias e para gêneros e espécies do Brasil. Mem. Inst. Oswaldo Cruz 2:121-129.

Barbosa P.B.B.M. 2005. Efeito sobre a participação de roedores na cadeia de transmissão de Leishmania infantum (Protozoa: Trypanossomatidae) no Rio Grande do Norte. Dissertação de Mestrado em Bioquímica, Universidade Federal do Rio Grande do Norte, Natal, RN. 85p.

Barbosa P.B.B.M., Queiroz P.V.S., Jerônimo S.M.B. \& Ximenes M.F.F.M. 2008. 
Experimental infection parameters in Galea spixii (Rodentia: Caviidae) with Leishmania infantum chagasi. Mem. Inst. Oswaldo Cruz 103:545548.

Barros-Battesti D.M., Arzua M. \& Bechara G.H. 2006. Carrapatos de Importância Médico-Veterinária da Região Neotropical: um guia ilustrado para identificação de espécies. Instituto Butantan, São Paulo. 223p.

Bittencourt E.B. \& Rocha C.F.D. 2002. Spatial use of rodents (Rodentia: Mammalia) host bodysurface by ectoparasites. Braz. J. Biol. 62:419-425.

Bukva V. 1985. Demodex flagellurus sp. n. (Acari: Demodicidae) from the preputial and clitoral glands of the house mouse, Mus musculus L. Folia Parasitol., Praha, 32:73-81.

Bukva V. 1994. Demodex agrarii sp. n. (Acari: Demodecidae) from cerumen and the sebaceous glands in the ears of the striped field mouse, Apodemus agrarius (Rodentia). Folia Parasitol., Praha, 41:305-311.

Bukva V. 1995. Demodex species (Acari: Demodecidae) parasitizing the brown rat, Rattus norvegicus (Rodentia): redescription of Demodex ratti and description of $D$. norvegicus sp.n. and D. ratticola sp.n. Folia Parasitol., Praha, 42:149-160.

Carvalho L.C.A., Pereira J.S., Sousa M.L.R., Oliveira M.F. \& Ahid S.M.M. 2010. Ocorrência de Ixodidae e Argasidae em Galea spixii spixii (Wagler, 1831), em Mossoró, Rio Grande do Norte. 6ํㅡㄹ Congresso Nordestino de Produção Animal, Mossoró, RN. (Resumo)

Hasegawa T. 1995. A case report of the management of demodicosis in the golden hamster. J. Vet. Med. Sci. 57:337-338.

Lacerda P.M.O., Moura C.E.B., Miglino M.A., Oliveira M.F. \& Albuquerque J.F.G. 2006. Origem do plexo lombossacral de mocó (Kerondon rupestris). Braz. J. Vet. Res. Anim. Sci. 43:620-628.
Lacher Jr T.E. 1981. The comparative social behavior of Kerodon rupestris and Galea spixii and the evolution of behavior in the Cavidae. Bull. Carnegie Museum Nat. Hist. 17:1-71.

Moojen J. 1952. Os Roedores do Brasil. Série A II. Biblioteca Científica Brasileira, Instituto Nacional do Livro, Ministério de Educação e da Saúde, Rio de Janeiro.

Nava S., Mangold A.J. \& Guglielmone A.A. 2006. The natural hosts of larvae and nymphs of Amblyomma tigrinum Koch, 1844 (Acari: Ixodidae). Vet. Parasitol. 140:124-132.

Nava S., Mangold A.J. \& Guglielmone A.A. 2008. Aspects of the life cycle of Amblyomma parvum (Acari: Ixodidae) under natural conditions. Vet. Parasitol. 156:270-276.

Nutting W.B. 1961. Demodex aurati sp. nov. and D. criceti, ectoparasites of the golden hamster (Mesocricetus auratus). Parasitol. 51:515-522.

Reis F.S., Barros M.C., Fraga E.C., Penha T.A., Teixeira W.C., Santos A.C.G. \& Guerra R.M.S.N.N. 2008. Ectoparasitos de pequenos mamíferos silvestres de áreas adjacentes ao rio Itapecuru e área de preservação ambiental do Inhamum, Estado do Maranhão, Brasil. Revta Bras. Parasitol. Vet. 17:69-74.

Schönfelder J., Henneveld K., Schönfelder A., Hein J. \& Müller R. 2010. Concurrent infestation of Demodex caviae and Chirodiscoides caviae in a guinea pig. Tierärztl. Praxis, Kleintiere, 38:28-30.

Werneck F.L. 1936. Contribuição ao conhecimento dos Mallophagos encontrados nos mamíferos sul-americanos. Mem. Inst. Oswaldo Cruz 31:391-589.

Werneck F.L. 1942. Sobre algumas espécies do gênero Gliricola (Mallophaga). Mem. Inst. Oswaldo Cruz 373:297-319. 